Research Article

\title{
The Prevalence of High Carcinogenic Risk of HPV Genotypes among HIV-Positive and HIV-Negative MSM from Russia
}

\author{
Ilia Beliakov $\mathbb{D}^{1},{ }^{1}$ Maria Senina $\mathbb{D}^{1},{ }^{1}$ Yuriy Tyulenev ${ }^{\mathbb{D}},{ }^{2}$ Elena Novoselova ${ }^{\mathbb{D}},{ }^{1}$ \\ Viktor Surovtsev, ${ }^{3}$ and Alexander Guschin $\left(\mathbb{D}^{4}\right.$ \\ ${ }^{1}$ NextBio Ltd. Co., Moscow 111394, Russia \\ ${ }^{2}$ InterLabService Ltd., Moscow 115035, Russia \\ ${ }^{3}$ RUDN University, Moscow 117198, Russia \\ ${ }^{4}$ Moscow Scientific and Practical Centre of Dermatovenerology and Cosmetology, Moscow 119071, Russia
}

Correspondence should be addressed to Ilia Beliakov; ibeliakov@gmail.com

Received 7 October 2020; Revised 19 April 2021; Accepted 22 May 2021; Published 2 June 2021

Academic Editor: Maria Lina Tornesello

Copyright (C) 2021 Ilia Beliakov et al. This is an open access article distributed under the Creative Commons Attribution License, which permits unrestricted use, distribution, and reproduction in any medium, provided the original work is properly cited.

Objective. Men who have sex with men (MSM) have a high risk of lifelong anal cancer caused by high-risk human papillomavirus (HR HPV) infections. The aim of this study was to investigate the prevalence of anal canal HR HPV infection among men who have sex with men (MSM) with and without HIV infection in Moscow (Russia). We evaluated associations of some HIV coinfections (HSV and CMV) and HPV distribution among MSM with and without HIV infection. Methods. Two groups of HIVpositive ( $n=60)$ and HIV-negative $(n=60)$ MSM were evaluated in the study. Fourteen high-risk (HR) HPV types, HSV1/2, and CMV were investigated in men anal swabs. Results. HR HPVs were found with nearly the same frequency of $66.7 \%$ in both groups: HIV-positive and HIV-negative MSM. HIV-positive status was statistically associated with the presence of several (more than two) HPV types $(p=0.044)$. The most prevalent HR HPV genotypes were HPV18, HPV16, HPV56, and HPV33 for HIV-positive MSM and HPV56, HPV51, HPV66, and HPV16 for HIV-negatives. We found a statistically significant association of five HR HPV types with HIV status of MSM: HPV16 $(p=0.028), \operatorname{HPV} 18(p=0.00006), \operatorname{HPV} 58(p=0.003), \operatorname{HPV} 33(p=0.019)$, and HPV39 $(p=0.026)$. The frequency of HSV1 (1.7\%) and HSV2 (10\%) infections and CMV (3.3\%) infection was evaluated in the group of HIV-positive MSM. The frequency of HSV1 (5\%) and HSV2 (6.7\%) infections and CMV (0\%) infection was evaluated, as well, in the group of HIV-negative MSM. Conclusion. Multiple HPV genotypes were detected significantly more often than single HPV genotype in the group of HIV-positive MSM. According to our data, 25\% of HIV-positive MSM have HPV39; this is the only one of the five types of $\operatorname{HR} \operatorname{HPV}(16,18,58,33$, and 39) associated with this group of MSM that has not yet been included in the HPV vaccines available on the market.

\section{Introduction}

Human papillomavirus (HPV) infection is the most common sexually transmitted infection (STI) worldwide. Mucosal HPV types from the alpha-genus infect squamous stratified epithelium from the mucosal tissues, and some infections may persist for many years with the potential to cause cancer of the cervix, vulva, vagina, penis, oropharynx, anus, and rectum $[1,2]$. The HPV-attributable anogenital cancer includes 8500 vulva, 12000 vagina 35000 anus (half occurring in men), and 13000 penis. Anal squamous cell cancer, biologically similar to cervical cancer, is rare, but its incidence is increasing [3]. Almost all cervical cancer and more than $80 \%$ of anal cancer are attributable to high-risk HPV (HR HPV) infection including HPV types 16, 18, 31, $33,35,39,45,51,52,56,58,59,68,73$, and $82[4]$ and are mainly associated with HPV16 and HPV18 $[5,6]$.

These high-risk genotypes of HPV were identified to be distributed with different prevalence in both women and men with lesions or abnormal cytology worldwide $[4,7,8]$. 
The prevalence of HPV infection in heterosexual men and MSM in the Russian population was reported previously [9-11]. Also, infection of HPV and HSV I/II was evaluated in people living with human immunodeficiency virus or having high-risk sexual behavior including MSM from Russia $[12,13]$. Comparison of HPV genotype prevalence and association with HIV status of MSM was previously reported in several populations $[8,14,15]$. However, there are no estimates of the prevalence of anal HPV and the distribution of high-risk HPV genotypes among HIV-negative and HIVpositive MSM from Russia [10, 11].

The HPV vaccines have been shown to be effective in preventing HPV infection and HPV-related low- and highgrade squamous intraepithelial lesions and cancer among both women and men $[16,17]$, and the 9-valent HPV vaccine has been shown to have a significantly increased potential impact compared with the quadrivalent vaccine among MSM [18]. The spectrum of high prevalent genotypes of $\mathrm{HPV}$ may be different in HIV-positive and HIV-negative MSM from Russia and influences the vaccine development $[10,11,13]$.

HPV vaccination (bi- and quadrivalent HPV vaccines) is currently approved only for women in Russia. MSM do not receive the same benefits as heterosexual men from the herd effect of immunity from HPV vaccinations in girls $[19,20]$. $\mathrm{HPV}$ vaccination is important among previously unvaccinated HIV-positive MSM aged up to and including 45 years [21-23].

\section{Methods}

2.1. Study Design. A cross-sectional study was conducted in Moscow, Russia, from November 2018 to October 2019. Participants were recruited by the nonprofit public organization "Step Fund" observed at the Moscow City Center for the Prevention and Control of AIDS and the Moscow Regional CDC. This study was approved by the Ethics Committee of the Institutional Review Board of the University of Peoples' Friendship University (RUDN University) of Russia, and written informed consent was obtained from each participant.

2.2. Data Collection. Participants were interviewed by welltrained health staff, and our special questionnaire was used to collect epidemiological data. Sociodemographic characteristics including age, past STIs (HPV and genital herpes), and CMV coinfection were collected. Participants were also asked about their behavioral characteristics including sexual orientation, sexual risks, and sexual behaviors.

2.3. Sample Collection and Laboratory Tests. Participants were asked to self-collect biomaterial from the anal canal using a regular flocked swab as described previously $[11,24]$ and store it in a universal transport media (NextBio, Russia) at $-20^{\circ} \mathrm{C}$.

Total DNA extraction was performed on $200 \mu \mathrm{l}$ of a sample using the MagnoPrime FAST kit (NextBio, IVD, Russia) on the Microlab STARLet platform (Hamilton Bonaduz AG, Switzerland) and eluted in $100 \mu \mathrm{l}$ TE buffer.
$10 \mu \mathrm{l}$ of each DNA sample was analyzed using the AmpliPrime HR HPV genotyping system (NextBio, IVD, Russia), allowing for the detection of 14 high cancer risk HPV genotypes (HPV 16, 18, 31, 33, 35, 39, 45, 51, 52, 56, 58, 59, 66, and 68).

HSV and CMV DNA detection was performed in DNA from anal swabs of MSM participants both with AmpliPrime HSV/CMV kit (NextBio, IVD, Russia) and with reference kit AmpliSens HSV I, II-FL (Central Research Institute of Epidemiology, IVD, Russia).

2.4. Statistical Analyses. Statistical analysis was evaluated with suitable statistical criteria. Fisher exact test and chisquared test were used to compare the distribution of categorical variables. A two-sided $p$ value $<0.05$ was considered statistically significant.

\section{Results}

3.1. Sociodemographic Characteristics. A total of $60 \mathrm{HIV}$ positive and $60 \mathrm{HIV}$-negative MSM participants were included in the study. The median age was 29 years (interquartile range (IQR), 22-33.5) for HIV-positives and 33 years (IQR: 26-34) for HIV-negatives (Table 1).

3.2. Sexual Orientation. The HIV-positive participants declared their sexual orientation as homosexual (73.3\%) and bisexual (26.6\%). The sexual orientation reported by HIVnegative MSM was $68.3 \%$ homosexual and $31.6 \%$ bisexual.

3.3. Prevalence of HPV Infection in HIV-Positive and HIVNegative MSM. Overall, the prevalence of high-risk HPV types was $73.3 \%$ among HIV-positive participants and $61.7 \%$ among HIV-negative men $(p=0.18)$ (Table 1$)$. We found that multiple HPV types were not significantly more frequent in the group of HIV-positive MSM $(p=0.2)$ HPV genotyping data are presented in Figure 1.

Asterisk marks the positive correlation of HPV genotype and HIV status. The Fisher exact test analysis of HPV genotyping data revealed the significant association of five HR HPV genotypes with the HIV-positive status of the participants (Figure 1): HPV16 $(p=0.028), \quad$ HPV18 $(p=0.00006), \operatorname{HPV} 58(p=0.003), \operatorname{HPV} 33(p=0.003)$, and HPV39 $(p=0.026)$. For other HPV genotypes, no significant correlations were shown.

3.4. Age and HR HPV Infection Depending on the HIV Status. We analyzed HPV infection prevalence in the two age groups: $<30$ years and $\geq 30$. Among our participants, the prevalence of anal HPV infection was the smallest within the 30- to 39-year interval (data not shown). The prevalence of any of HR HPV among HIV-positive participants was not significantly different: $44.2 \%$ (19/43) among those under 30 years old versus $56.8 \%(21 / 37)$ among the participants over 30 years $(p=0.27)$ and increased with age similar to HIV infection: $45.1 \%$ (MSM under 30 ) vs. $57.1 \%$ (MSM over 30 ) (Table 1). 
TABLE 1: Characteristics of the study population $(n=120)$ of HIV-positive and HIV-negative MSM from Russia (2016-2017).

\begin{tabular}{|c|c|c|c|c|c|c|c|c|}
\hline \multirow[t]{2}{*}{ Influence of risk factors on HIV-infection } & & \multicolumn{2}{|c|}{$\begin{array}{c}\text { Total } \\
(n=120)\end{array}$} & \multicolumn{2}{|c|}{$\begin{array}{l}\text { HIV-negative } \\
\quad(n=60)\end{array}$} & \multicolumn{2}{|c|}{$\begin{array}{l}\text { HIV-positive } \\
\quad(n=60)\end{array}$} & \multirow[t]{2}{*}{$p$ value } \\
\hline & & No. & $\%$ & No. & $\%$ & No. & $\%$ & \\
\hline \multicolumn{9}{|l|}{ Demographic characteristics } \\
\hline Median age (IRQ) $)^{\#}$ & & 30 & $-34)$ & & $-34)$ & 29 & 33.5) & \multirow[b]{2}{*}{0.2} \\
\hline Age group & $\begin{array}{l}<30 \\
\geq 30\end{array}$ & $\begin{array}{l}71 \\
49\end{array}$ & $\begin{array}{l}59.2 \\
40.8\end{array}$ & $\begin{array}{l}39 \\
21\end{array}$ & $\begin{array}{l}65 \\
35\end{array}$ & $\begin{array}{l}32 \\
28\end{array}$ & $\begin{array}{l}53.3 \\
46.7\end{array}$ & \\
\hline \multicolumn{9}{|l|}{$\begin{array}{l}\text { Sexual behavior } \\
\text { No. of lifetime male sex partners }\end{array}$} \\
\hline No. of lifetime male sex partners & $\begin{array}{c}<100 \\
100-500 \\
>500\end{array}$ & $\begin{array}{c}47 \\
64 \\
9 \\
\end{array}$ & $\begin{array}{c}41 \\
54 \\
9 \\
\end{array}$ & $\begin{array}{c}26 \\
32 \\
2 \\
\end{array}$ & $\begin{array}{c}47 \\
50 \\
8 \\
\end{array}$ & $\begin{array}{c}21 \\
32 \\
7 \\
\end{array}$ & $\begin{array}{l}35 \\
58 \\
10 \\
\end{array}$ & $\begin{array}{l}0.16 \\
0.38 \\
0.69 \\
\end{array}$ \\
\hline \multicolumn{9}{|l|}{ Median no. of anal sex partners last 6 months } \\
\hline No. of anal sex partners last 6 months & $\begin{array}{l}\leq 1 \\
2-5 \\
\geq 6\end{array}$ & $\begin{array}{l}22 \\
46 \\
52\end{array}$ & $\begin{array}{l}18 \\
38 \\
43\end{array}$ & $\begin{array}{l}10 \\
25 \\
25\end{array}$ & $\begin{array}{l}17 \\
42 \\
42\end{array}$ & $\begin{array}{l}12 \\
21 \\
27\end{array}$ & $\begin{array}{l}20 \\
35 \\
45\end{array}$ & $\begin{array}{l}0.35 \\
0.46 \\
0.71\end{array}$ \\
\hline \multicolumn{9}{|l|}{ Study results } \\
\hline HPV infection vs HPV-negative & & 80 & 66.7 & 40 & 66.7 & 40 & 66.7 & 1 \\
\hline Multiple ${ }^{* *}$ HPV infection & & 60 & 50 & 34 & 56.7 & 26 & 43.3 & \multirow{2}{*}{$0.044^{*}$} \\
\hline Single HPV infection & & 20 & 16.7 & 6 & 10 & 14 & 23.3 & \\
\hline HPV16 & & 27 & 22.5 & 8 & 13.3 & 19 & 31.7 & $0.028^{*}$ \\
\hline HPV18 & & 23 & 19.2 & 3 & 5 & 20 & 33.3 & $0.00006^{*}$ \\
\hline HPV58 & & 13 & 10.8 & 2 & 3.3 & 11 & 18.3 & $0.003^{*}$ \\
\hline HPV33 & & 18 & 15 & 5 & 8.3 & 13 & 21.7 & $0.019^{*}$ \\
\hline HPV39 & & 15 & 12.5 & 4 & 6.7 & 11 & 18.3 & $0.026^{*}$ \\
\hline HIV-positive MSM within age groups & $\begin{array}{l}<30 \\
\geq 30\end{array}$ & $\begin{array}{l}71 \\
49\end{array}$ & $\begin{array}{l}59.2 \\
40.8\end{array}$ & & & & & \multirow[b]{2}{*}{0.27} \\
\hline Any HR HPV-positive within age groups & $<30$ & 43 & $\begin{array}{l}70.0 \\
70.4 \\
63.3\end{array}$ & $\begin{array}{l}24 \\
16\end{array}$ & $\begin{array}{l}55.8 \\
43.2\end{array}$ & $\begin{array}{l}19 \\
21\end{array}$ & $\begin{array}{l}44.2 \\
56.8\end{array}$ & \\
\hline \multicolumn{9}{|l|}{ Co-infections } \\
\hline HSV & & 14 & 11.7 & 7 & 11.7 & 7 & 11.7 & 1 \\
\hline HSV I & & 4 & 3.3 & 3 & 5 & 1 & 1.7 & 0.37 \\
\hline HSV II & & 10 & 8.3 & 4 & 6.7 & 6 & 10 & 0.5 \\
\hline CMV & & 2 & 1.7 & 0 & 0 & 2 & 3.3 & - \\
\hline
\end{tabular}

IQR: interquartile range. ${ }^{*} \mathrm{~A}$ two-sided $p$ value $<0.05$ was considered statistically significant; ${ }^{* *}$ multiple HPV: two and more HPV types.

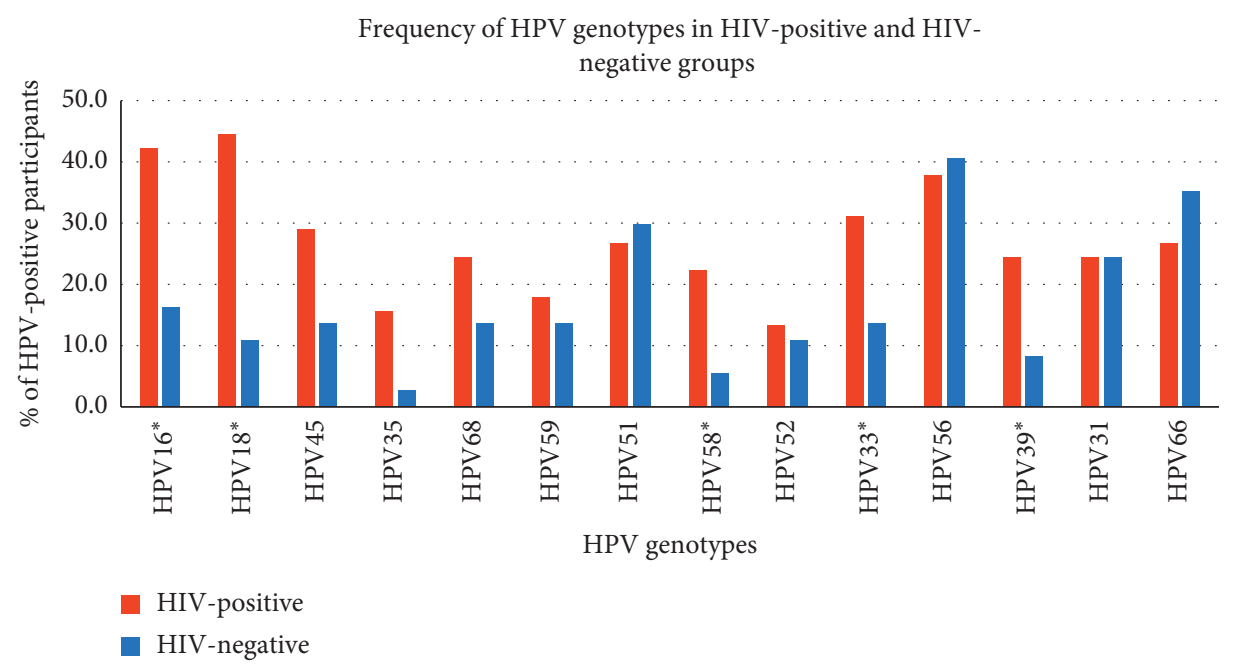

FIgURe 1: HPV genotypes in HIV-positive and HIV-negative MSM $(N=120)$. Asterisk marks the significant correlation of HPV genotype and HIV status. 
3.5. HSV and CMV Testing. HSV I/II was found in $11.7 \%$ (7/ 60) of HIV-positive participants (HSV I: 1/60, 1.7\%; HSV II: $6 / 60,10 \%)$ and in $11.7 \%$ (7/60) of HIV-negative (HSV I: $3 /$ 60, 5\%; HSV II: 4/60, 6.7\%). CMV DNA was identified in two HIV-positive participants $(2 / 60,3.3 \%)$, and no CMV was detected in HIV-negative participants.

\section{Discussion}

In this study, we showed the prevalence of anal HPV infection, genotype distribution, and the risk factors associated with the infection among MSM with or without HIV from Moscow, Russia. The average age in the two groups of our study was nearly equal (29.8 vs. 28.7 years). This study results confirmed data obtained from similar observations on the MSM population from Holland [25] and Russia [11]-HIV infection correlates with HPV infection.

HSV and CMV are known as the most frequent opportunistic infections among HIV-positive people, especially among MSM. It was previously shown by different authors HSV I/II and CMV coinfection in HIV-positive and HIV-negative MSM $[26,27]$. Our data revealed a lower rate of HSV 1 coinfection in HIV-positive MSM in comparison with those in HIV-negative MSM (HSV1: 1.7\% vs. $5 \%$ ), and CMV was detected only in the HIV-positive group.

On the other side, the HIV-positive MSM tended to have more sexual partners and more sex episodes per lifetime than HIV-negative MSM [25]. We observed nearly equal sexual activity with both criteria: "a number of anal sex partners in the last 6 months" and "a number of lifetime male sex partners" in HIV-positive and HIV-negative groups. No significant correlation in sexual activity of MSM was observed in HIV-positive and HIV-negative groups. That might be due to the small number of participants in our study. It should be noted that the study participants had no health complaints at the moment of data and sample collection while CMV infections in the colon are common in HIV-infected people, often accompanied by anal ulcers in MSM.

It was found that HPV infection was associated with MSM and was detected with a high frequency $(67.7 \%)$ regardless of HIV status. The most prevalent HR HPV genotypes reported in this study were HPV18 (33.3\%), HPV16 (31.7\%), HPV56 (28.3\%), and HPV33 (21.7\%) for HIVpositive MSM and HPV51 (20\%), HPV56 (23.3\%), and HPV66 (18.3\%) for HIV-negative MSM. HPV16/18 previously showed $31.7 \%$ among MSM [11] compared to our data (40.8\% (49/120) of all MSM tested). HPV16/18 were detected in the HIV-negative MSM group in $16.7 \%$ in the present study vs. $23 \%$ in the Wirtz study, and in the HIVpositive group, HPV16/18 were observed in $65 \%$ in our study vs. $41.4 \%$ in the Wirtz study [11].

We revealed association of five high-risk HPV genotypes with HIV status of MSM: HPV16 $(p=0.028)$, HPV18 $(p=0.00006)$, HPV58 $(p=0.003), \operatorname{HPV} 33(p=0.019)$, and HPV39 $(p=0.026)$. One of the reasons for the correlation between the detection of HPV coinfection as previously published was the lower rate of HPV clearance in HIVpositive men $[28,29]$.

The higher rate of HPV associated with anal cancer incidence worldwide was observed among HIV-positive MSM (46 per 100,000 per year) compared to HIV-negative MSM (5 per 100,000 per year) [8, 24, 28].

There were several limitations to the current study. First of all, the selection of the study participants was not random. Due to the limitation of time of the study, we restricted the number of participants to $60 \mathrm{HIV}$-positive MSM and also randomly chose an equal number of participants from a bigger HIV-negative group. Second, the risk behaviors were measured based on self-report, which might lead to information bias. Third, although health professionals were trained using a standard protocol, the self-collection of anal canal cell samples might not be completely successful, and the test kit for HPV included 14 genotypes, which might together lead to an underestimation of the prevalence.

Cancer prevention of HIV-positive MSM highly depends on HR HPV vaccination. The majority of anal cancer cases associated with HPV 16/18 types were underrepresented in HIV-positive MSM diagnosed with anal cancers. Only several or few studies were published for now about HPV prevalence in anal cancer among HIV-positive MSM (HPV16: 67\%; HPV18: 24\%; HPV 31/33/45/52/58: 26\%) and more thorough investigations of HPV genotypes should be performed on MSM anal cancers [15, 30]. HPV16/18-positive, HIV-positive MSM were detected in $48.3 \%$ while HPV16/18-positive, HIVnegative MSM were detected in only $20 \%$ in our study. Probably, HPV unvaccinated MSM more frequently coinfected both HPV and HIV. For the current vaccines present on the market, the nine-valent vaccine can protect from nearly $100 \%$ of described previously HR HPV in anal cancers of MSM (for cardasil9: 16, 18, 31, 33, 45, 52, and 58). We revealed a statistically significant association of five HR HPVs infections with HIV-positive MSM: HPV16, HPV18, HPV58, HPV33, and HPV39 in this study population.

HPV vaccination is not approved for men in Russia [16-18]: the 4-valent Gardasil (HPV 6, 11, 16, and 18) (Merck) vaccine is routinely prescribed for women vaccination and the nine-valent vaccine is not yet approved in Russia. The only HPV39 was not included in vaccines available on the market. According to recent data, about 7\% of HIV-positive anal carcinomas contain HPV39 genotype [30]. This is an important issue that requires further detailed study: HPV vaccination in young and old MSM for the prevention of cancer associated with HPV [19] in Russia.

\section{Abbreviations}

HR HPV: High-risk human papillomavirus

MSM: $\quad$ Men who have sex with men

STI: $\quad$ Sexually transmitted infection

IVD: In vitro diagnostic

HSV: $\quad$ Herpes simplex virus

HIV: Human immunodeficiency virus

CMV: Cytomegalovirus

AIN: Anal intraepithelial neoplasia. 


\section{Data Availability}

The data used to support the findings of this study are available from the corresponding author upon request.

\section{Ethical Approval}

The authors assert that all procedures contributing to this work comply with the ethical standards of the relevant national and institutional committees on human experimentation and with the Helsinki Declaration of 1975, as revised in 2008 .

\section{Conflicts of Interest}

The authors declare that they have no conflicts of interest.

\section{Acknowledgments}

The authors thank all the participants for their contribution to the study. This study was supported by the Ministry of Education and Science of the Russian Federation, project no. 03.G25.31.0226, March 3, 2017.

\section{References}

[1] H. zur Hausen, "Human papillomaviruses in the pathogenesis of anogenital cancer," Virology, vol. 184, no. 9, pp. 9-13, 1991.

[2] E. Schwarz, U. K. Freese, L. Gissmann et al., "Structure and transcription of human papillomavirus sequences in cervical carcinoma cells," Nature, vol. 314, no. 3, pp. 111-114, 1985.

[3] C. de Martel, M. Plummer, J. Vignat, and S. Franceschi, "Worldwide burden of cancer attributable to HPV by site, country and HPV type," International Journal of Cancer, vol. 141, no. 8, pp. 664-670, 2017.

[4] M. Arbyn, M. Tommasino, C. Depuydt, and J. Dillner, "Are 20 human papillomavirus types causing cervical cancer?" The Journal of Pathology, vol. 234, no. 12, pp. 431-435, 2014.

[5] B. E. Hoots, J. M. Palefsky, J. M. Pimenta, and J. S. Smith, "Human papillomavirus type distribution in anal cancer and anal intraepithelial lesions," International Journal of Cancer, vol. 124, no. 5, pp. 2375-2383, 2009.

[6] M. Plummer et al., "Global burden of cancers attributable to infections in 2012: a synthetic analysis," The Lancet Global Health, vol. 4, pp. e609-e616, 2016.

[7] M. Arbyn, A. Anttila, J. Jordan et al., "European guidelines for quality assurance in cervical cancer screening. Second editionsummary document," Annals of Oncology, vol. 21, no. 3, pp. 448-458, 2010.

[8] D. A. Machalek, M. Poynten, F. Jin et al., "Anal human papillomavirus infection and associated neoplastic lesions in men who have sex with men: a systematic review and metaanalysis," The Lancet Oncology, vol. 13, no. 5, pp. 487-500, 2012.

[9] V. Smelov, K. M. Elfström, C. Eklund, O. Sokolova, and J. Dillner, "Determinants of the presence of human papillomaviruses in the anal canal of Russian men," Journal of Medical Virology, vol. 90, no. 10, pp. 1643-1650, 2018.

[10] V. Smelov, R. Muwonge, O. Sokolova et al., "Beta and gamma human papillomaviruses in anal and genital sites among men: prevalence and determinants," Scientific Reports, vol. 8, no. 5, p. 8241,2018 .
[11] A. L. Wirtz, C. E. Zelaya, A. Peryshkina et al., "Anal human papillomavirus and HIV: a cross-sectional study among men who have sex with men in Moscow, Russia, 2012-2013," Eurosurveillance, vol. 20, no. 4, 2015.

[12] Y. A. Amirkhanian, J. A. Kelly, A. A. Kukharsky et al., "Predictors of HIV risk behavior among Russian men who have sex with men: an emerging epidemic," AIDS-Acquired Immuno Deficiency Syndrome, vol. 15, no. 3, pp. 407-412, 2001.

[13] S. Baral, D. Kizub, N. F. Masenior et al., "Male sex workers in Moscow, Russia: a pilot study of demographics, substance use patterns, and prevalence of HIV-1 and sexually transmitted infections," AIDS Care, vol. 22, no. 1, pp. 112-118, 2010.

[14] X. Liu, H. Lin, X. Chen et al., "Prevalence and genotypes of anal human papillomavirus infection among HIV-positive vs. HIV-negative men in Taizhou, China," Epidemiology and Infection, vol. 147, no. 1, p. e117, 2019.

[15] C. de Martel, M. S. Shiels, S. Franceschi et al., "Cancers attributable to infections among adults with HIV in the United States," AIDS-Acquired Immuno Deficiency Syndrome, vol. 29, no. 10, pp. 2173-2181, 2018.

[16] M. Arbyn, L. Xu, C. Simoens, and P. P. L. Martin-Hirsch, "Prophylactic vaccination against human papillomaviruses to prevent cervical cancer and its precursors," Cochrane Database of Systematic Reviews, vol. 5, no. 5, Article ID CD009069, 2018.

[17] E. J. Crosbie, M. H. Einstein, S. Franceschi, and H. C. Kitchener, "Human papillomavirus and cervical cancer," Lancet, vol. 382, no. 7, pp. 889-899, 2013.

[18] A. S. Forster and R. Gilson, "Challenges to optimising uptake and delivery of a HPV vaccination programme for men who have sex with men," Human Vaccines \& Immunotherapeutics, vol. 15, pp. 1541-1543, 2019.

[19] M. Drolet, É. Bénard, N. Pérez, and M. Brisson, "HPV Vaccination Impact Study Group. Population-level impact and herd effects following the introduction of human papillomavirus vaccination programmes: updated systematic review and meta-analysis," Lancet, vol. 394, no. 10, pp. 497509, 2019.

[20] G. L. Mortensen, "Drivers and barriers to acceptance of human-papillomavirus vaccination among young women: a qualitative and quantitative study," BMC Public Health, vol. 10 , no. 2 , p. $68,2010$.

[21] M. A. Gerend, K. Madkins, G. Phillips, and B. Mustanski, "Predictors of human papillomavirus vaccination among young men who have sex with men," Sexually Transmitted Diseases, vol. 43, no. 3, pp. 185-191, 2016.

[22] A. Lin, K. J. Ong, P. Hobbelen et al., "Impact and cost-effectiveness of selective human papillomavirus vaccination of men who have sex with men," Clinical Infectious Diseases, vol. 64, no. 5, pp. 580-588, 2017.

[23] Joint Committee on Vaccination and Immunisation, JCVI statement on HPV vaccination of men who have sex with men 2015 [Available from: 2015, https://www.gov.uk/government/ publications/jcvi-statement-on-hpv-vaccination-of-men-whohave-sex-with-men].

[24] M. K. L. Poon, J. P. H. Wong, A. T. W. Li et al., "HIV-positive MSM's knowledge of HPV and anal cancer self-sampling: a scoping review," Current Oncology, vol. 25, no. 2, pp. e83-e89, 2018.

[25] E. Marra, A. King, E. van Logchem et al., "Anal HPV 16 and 18 viral load: a comparison between HIV-negative and -positive MSM and association with persistence," Journal of Medical Virology, vol. 90, no. 1, pp. 76-83, 2018. 
[26] W. Borena, S. Kruis, M. Kitchen et al., "Anal Ureaplasma spp. positivity among HIV positive men who have sex with men may be associated with high-risk-type HPV infections," International Journal of Infectious Diseases, vol. 84, no. 7, pp. 75-79, 2019.

[27] L. E. Nelson, W. Tharao, W. Husbands et al., "The epidemiology of HIV and other sexually transmitted infections in African, Caribbean and Black men in Toronto, Canada," BMC Infectious Diseases, vol. 19, no. 3, p. 294, 2019.

[28] L. Darwich, S. Videla, M. P. Cañadas et al., "HIV-HPV Team, Distribution of human papillomavirus genotypes in anal cytological and histological specimens from HIV-infected men who have sex with men and men who have sex with women," Diseases of the Colon \& Rectum, vol. 56, no. 9, pp. 1043-1052, 2013.

[29] P. Patel, T. Bush, E. M. Kojic et al., "Prevalence, incidence, and clearance of anal high-risk human papillomavirus infection among HIV-infected men in the SUN study," The Journal of Infectious Diseases, vol. 217, no. 6, pp. 953-963, 2018.

[30] C. Lin, S. Franceschi, and G. M. Clifford, "Human papillomavirus types from infection to cancer in the anus, according to sex and HIV status: a systematic review and meta-analysis," The Lancet Infectious Diseases, vol. 18, no. 2, pp. 198-206, 2018. 